\title{
Bioanalysis
}

\section{Method development of glycoprotein biomarkers for cancers}

\author{
“...development of a biomarker needs to consider how to \\ implement in clinical trials based on FDA requirements and \\ regulations."
}

First draft submitted: 12 April 2017; Accepted for publication: 3 May 2017;

Published online: 23 June 2017

Keywords: chemoenzymatic $\bullet$ chromatography $\bullet$ glycomics $\bullet$ glycoproteomics $\bullet$ liquid chromatography $\bullet$ mass spectrometry $\bullet$ solid-phase

\section{Overview of progress in glycoprotein biomarker}

Cancer biomarkers are substances that are produced by tumor or other cells in response to benign or malignant conditions. They are often produced at much higher levels in cancerous conditions. Some specific biomarkers may be associated with only one type of cancer, whereas others are found in two or more cancer types. Biomarkers are used to help detect, diagnose and manage certain types of cancer. As a result, tremendous efforts have been devoted to characterize upregulated proteins or genes in specimens from cancerous patients. Although an elevated level of a biomarker may suggest the presence of cancer, this alone is not enough to diagnose cancer. Therefore, measurements of biomarkers may be combined with other tests, such as biopsies, to diagnose cancer [1].

Glycoproteins have emerged as one of the promising targets in development of disease biomarkers for diagnosis and prognosis. Protein glycosylation is synthesized and modified within two membrane-bound organelles in the cell: the rough endoplasmic reticulum and the Golgi apparatus [2]. The aberrant glycosylation may be developed in abnormal cells. Monitoring of glycosylation can potentially indicate the status of physiology of living organisms. For instance, total prostate-specific antigen (PSA) has been utilized for diagnosis and monitoring of prostate cancer since 1986 [3]. The PSA is an N-glycosylated protein that is found in human prostate tissue, serum and urine [4].
The presence of the PSA in serum and urine is used as a biomarker for the occurrence of prostate cancer. Another glycoprotein, $\alpha(1,6)-$ fucosyltransferase (FUT8) is substantially overexpressed in aggressive prostate cancer, whereas PSA remains the same in aggressive or nonaggressive phenotypes $[5,6]$. Lung cancer is by far the leading cause of cancer death among men and women [7]. Mutations of $E G F$ receptor, a highly glycosylated protein, in lung cancer are correlated with clinical response to Gefitinib therapy. EGF receptor mutation testing helps guide treatment and determine whether patients with non-small-cell lung cancer may benefit from targeted therapy [8]. Overall, discovery of the potential glycoprotein biomarkers is accomplished by comparing the glycoprotein profiles between tumor and normal tissues or biofluids $[9,10]$. Since tumor biomarkers are associated with diseases, they can be used for diagnosis, prognosis and prediction [11].

\section{Why glycoproteins are ideal targets for biomarker development}

A biomarker could be used for early detection and monitoring the course of disease progression or the patient's response to the particular therapy. An ideal biomarker needs to consider the specific application of a target. For early detection or monitoring, it is ideal to use noninvasive approaches to collect samples from patients, such as serum, saliva, bronchoalveolar lavage or urine. Studies have shown most pro-

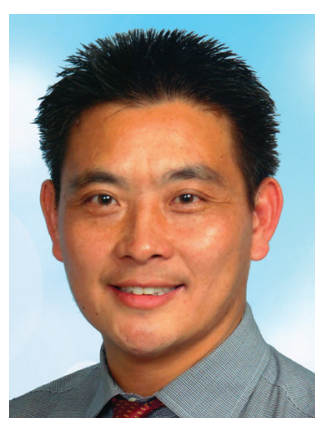

Shuang Yang

Laboratory of Bacterial Polysaccharides, Center for Biological Evaluation and Research, US FDA, Silver Spring, MD 20993, USA

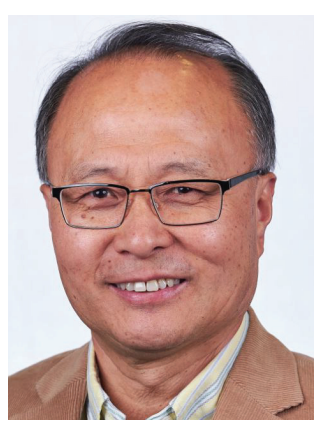

Perry G Wang

Author for correspondence: Office of Regulatory Science, CFSAN, US FDA, College Park, MD 20740, USA perry.wang@fda.hhs.gov

newlands press part of 
teins in human biofluids are glycosylated, especially for extracellular matrix and membrane proteins $[12,13]$. These highly abundant glycoproteins probably represent physiological stage of a living cell or organism. Glycoproteins are those proteins that are covalently linked by glycans (or carbohydrates) to their asparagine or serine/threonine. These conjugated proteins are represented by many substances of biological importance, such as critical roles in human diseases by affecting activities of enzymes, hormones and antibodies [14]. After glycosylation was redefined in later 1980s, $\mathrm{N}$-glycans and O-glycans were discovered in which they conjugate to different amino acids of proteins. It was found that the biosynthesis of $\mathrm{N}$ - and O-glycan is controlled at the level of gene expression, mRNA, enzyme protein activity and localization, and through substrate and cofactor concentration at the site of synthesis. The glycosylation can be disease specific and thus is associated with the state of the disease [15]. For example, patients with occurrence of tumor often have elevated levels of serum proteins that are highly sialylated, but their glycoprotein levels are decreased when patients received chemotherapy [16]. Numerous studies have dem- onstrated that glycoproteins in serum, urine and saliva are useful biomarkers for diagnosis and therapeutic monitoring [17]. Furthermore, the identified biomarkers can be utilized as surrogate end points in mechanism-driven oncology drug development [18].

\section{Methods that specialize for selection of glycoprotein}

Glycosylation is complex due to a great deal of oligosaccharides, types of glycans conjugated with amino acids and glycosite occupancy. Many methods have been developed for enrichment of glycoproteins via chemical conjugation/labeling or affinity interaction.

Chemical conjugation has been widely employed for enrichment of glycosite-containing glycoproteins since it was established in 2003 [19,20]. It utilizes the glycans that are linked to asparagine in the motif of asparagine$\mathrm{X}$-serine/threonine (where $\mathrm{X}$ is any amino acid except for proline). Using sodium periodate $(>10 \mathrm{mM})$ at room temperature enables to oxidize most cis-diols of glycans, resulting in enrichment of N-linked and O-linked glycopeptides (Figure 1); when a low amount of sodium

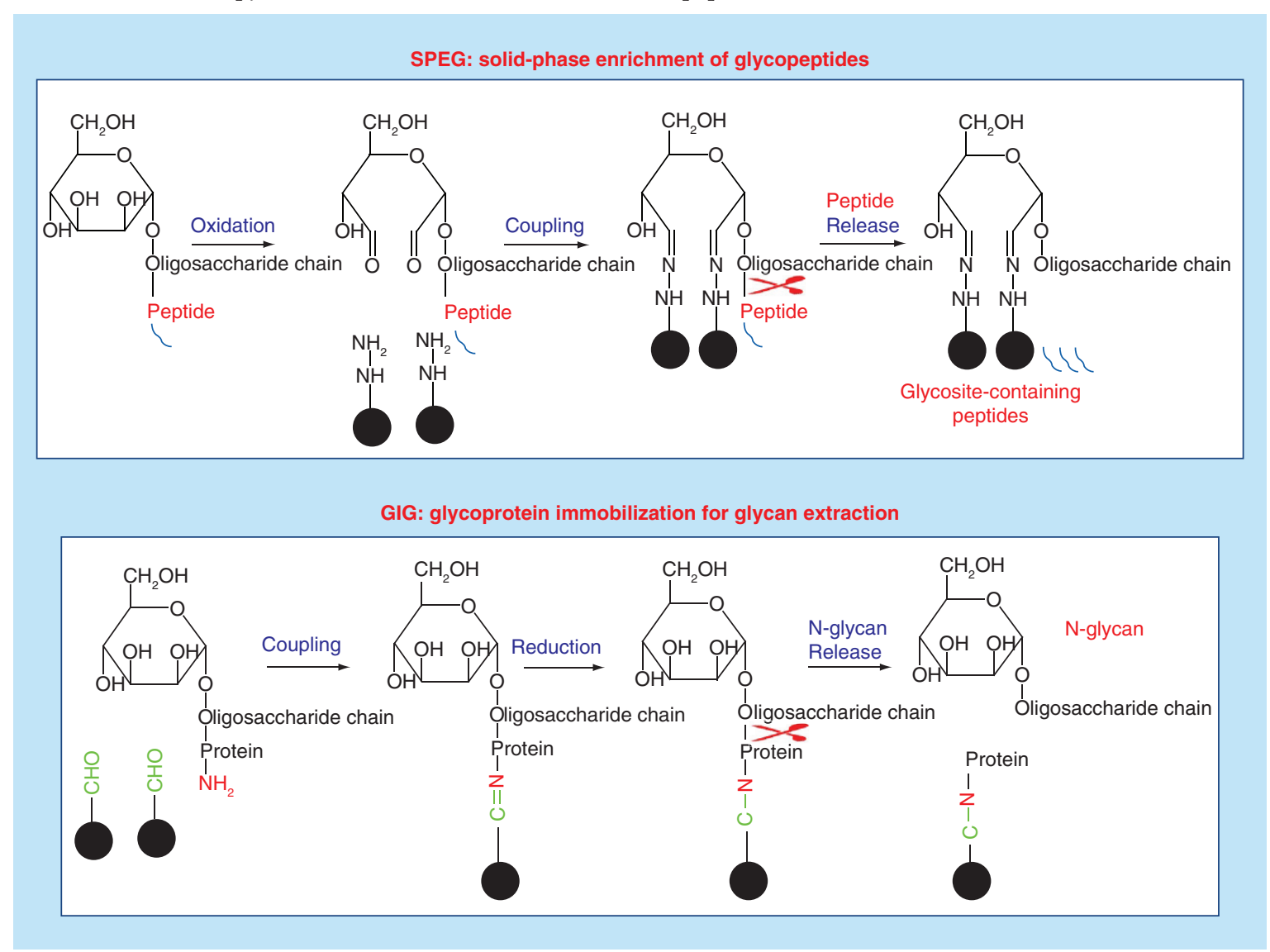

Figure 1. Schematic diagram of enrichment of glycosite-containing peptides and glycans by chemical conjugation on the solid support. SPEG oxidizes cis-diols of carbohydrates for specific conjugation of glycopeptides [19]; GIG immobilizes lysines or $\mathrm{N}$-termini via reductive amination.

Adapted with permission from [19] @ Macmillan Publishers Ltd: Nature Biotechnology (2003) and [24] @ Nature Publishing Group; American Chemical Society (2013). 
periodate $(1 \mathrm{mM})$ at $4^{\circ} \mathrm{C}$, only sialic acids are specifically oxidized for conjugation via hydrazide chemistry. Glycoproteins can also be enriched by the reaction with boronic acid, forming stable boronic diesters under basic conditions [21]. These techniques are useful as targeted proteomic strategies for clinical biomarker discovery in human plasma, tissue and urine [22,23].

Glycans were recently explored for disease biomarkers since glycan landscape is often a hallmark of disease states. Cancer cells frequently show glycans at different levels or with fundamentally different structures than those detected on normal cells [25]. For example, $\mathrm{N}$-glycan profiling on the colorectal cancer cells and tissues shows a strong representation of high mannose and $\alpha 2,6$-linked sialylated complex $\mathrm{N}$-glycans, whereas metastatic and aggressive cells have unique bisecting $\beta 1,4-G l c N A c y l a t i o n$ and $\alpha 2,3$-linked sialylation [26]. Glycan analysis has been routinely performed by enzymatic digestion and chemical reaction in solution. To identify detailed structures, glycans are permethylated by replacing $-\mathrm{OH}$ with $-\mathrm{OCH}_{3}$. Recently, a chemoenzymatic solid-phase method has been developed for sequential release of $\mathrm{N}$-glycans and $\mathrm{O}$-glycans [24,27]. The method (glycoprotein immobilization for glycan extraction) uses lysines or $\mathrm{N}$-termini for conjugation on resin that functionalizes with aldehyde groups (Figure 1). Glycans can be chemically or enzymatically modified on resin and later released by PNGase F, followed by reaction in ammonia in the presence of 1-phenyl3-methyl-5-pyrazolone. An HPLC-based 96-well plate platform has also been reported for high-throughput analysis of glycans [28].

Lectins are carbohydrate-binding proteins that capture oligosaccharide epitopes on glycoproteins. Each lectin has a relatively broad specificity on different types of glycoproteins, such as concanvavalin A, preferentially binding to mannose and glucose residues, sambucus nigra lectin-I binding to $\alpha 2,6$-linked sialic acid residue and maackia amurensis lectin binding to $\alpha 2,3-$ linked sialic acid residue. Using lectin-based strategies, selective enrichment of glycoproteins from serum or plasma is implemented for cancer biomarker discovery [29,30]. In addition, glycopeptide enrichment can also be achieved by hydrophilic interaction liquid chromatograph through polar-polar interaction [1,31]. Recently, a platform using hydrophilic

\section{References}

1 Taylor DD, Gercel-Taylor C. MicroRNA signatures of tumor-derived exosomes as diagnostic biomarkers of ovarian cancer. Gynecol. Oncol. 110(1), 13-21 (2008).

2 Moremen KW, Tiemeyer M, Nairn AV. Vertebrate protein glycosylation: diversity, synthesis and function. Nat. Rev. Mol. Cell Biol. 13(7), 448-462 (2012). interaction liquid chromatography-MS/MS has been developed for in vitro skin-penetration studies [32], which has potential in biomarker discovery for skin cancers.

\section{Challenge \& future perspective}

It becomes a trend to use protein signatures, biology markers and clinical-pathological features as the personalized therapy recently. Often these approaches present a great deal of challenges, partially due to the imperfect design of experiments for biomarker discovery [32]. Second, the window between when early detection can improve outcome and when it becomes too late for effective intervention is often narrow; sensitivity and specificity are critical aspects for accurate detection; a validated biomarker may result in test-negative individuals or vice versa [33]. Third, there are varied approaches that have been widely accepted for profiling of cancer glycoproteome in its various dimensions; it is thus impractical to explore cancer-related biomarkers by any individual approach, not to mention a wide range of tissue heterogeneity. It becomes convincing to incorporate a comprehensive human proteome, as well as posttranslational modification of proteome, for a large-scale effort to elucidate the range of glycoproteome alterations in cancer. The national and international collaboration on this regard may advance the quest to decipher and identify biomarkers, such as the project by the Clinical Proteomic Tumor Analysis Consortium [34]. Moreover, it is nontrivial to translate those discoveries into the US FDA-approved biomarkers. It takes tremendous efforts to have biomarker from lab-bench to bed-side, as well as being approved by the FDA. Thus, development of a biomarker needs to consider how to implement in clinical trials based on FDA requirements and regulations.

\section{Financial \& competing interests disclosure}

The authors, P Wang and S Yang, are both employees of the US FDA. The authors have no other relevant affiliations or financial involvement with any organization or entity with a financial interest in or financial conflict with the subject matter or materials discussed in the manuscript. This includes employment, consultancies, honoraria, stock ownership or options, expert testimony, grants or patents received or pending, or royalties.

No writing assistance was utilized in the production of this manuscript.

3 Thompson IM, Pauler DK, Goodman PJ et al. Prevalence of prostate cancer among men with a prostate-specific antigen level $\leq 4.0 \mathrm{ng}$ per milliliter. N. Engl. J. Med. 350(22), 2239-2246 (2004).

4 Oesterling JE. Prostate specific antigen: a critical assessment of the most useful tumor marker for adenocarcinoma of the prostate. J. Urol. 145(5), 907-923 (1991). 
5 Wang X, Chen J, Li QK et al. Overexpression of $\alpha(1,6)$ fucosyltransferase associated with aggressive prostate cancer. Glycobiology 24(10), 935-944 (2014).

6 Shah P, Wang X, Yang W et al. Integrated proteomic and glycoproteomic analyses of prostate cancer cells reveal glycoprotein alteration in protein abundance and glycosylation. Mol. Cell Proteomics 14(10), 2753-2763 (2015).

7 Siegel RL, Miller KD, Jemal A. Cancer statistics, 2015. CA Cancer J. Clin. 65(1), 5-29 (2015).

8 Paez JG, Jänne PA, Lee JC et al. EGFR mutations in lung cancer: correlation with clinical response to gefitinib therapy. Science 304(5676), 1497-1500 (2004).

9 Grønborg M, Kristiansen TZ, Iwahori A et al. Biomarker discovery from pancreatic cancer secretome using a differential proteomic approach. Mol. Cell Proteomics 5(1), 157-171 (2006).

10 Block TM, Comunale MA, Lowman M et al. Use of targeted glycoproteomics to identify serum glycoproteins that correlate with liver cancer in woodchucks and humans. Proc. Natl Acad. Sci. USA 102(3), 779-784 (2005).

11 Ciocca DR, Calderwood SK. Heat shock proteins in cancer: diagnostic, prognostic, predictive, and treatment implications. Cell Stress Chaperon 10(2), 86-103 (2005).

12 Alberts B, Johnson A, Lewis J, Raff M, Roberts K, Walter P. The extracellular matrix of animals. In: Molecular Biology of the Cell. Garland Science, NY, USA, 1616 (2002).

13 Spiro RG. Protein glycosylation: nature, distribution, enzymatic formation, and disease implications of glycopeptide bonds. Glycobiology 12(4), R43-R56 (2002).

14 Spiro RG. Glycoproteins: their biochemistry, biology and role in human disease. N. Eng. J. Med. 281(18), 991-1001 (1969).

15 Brockhausen I, Schutzbach J, Kuhns W. Glycoproteins and their relationship to human disease. Cells Tissues Organs 161(1-4), 36-78 (1998).

16 Lipton A, Harvey HA, Delong S et al. Glycoproteins and human cancer: 1. Circulating levels in cancer serum. Cancer 43(5), 1766-1771 (1979).

17 Durand G, Seta N. Protein glycosylation and diseases: blood and urinary oligosaccharides as markers for diagnosis and therapeutic monitoring. Clin. Chem. 46(6), 795-805 (2000).

18 Park JW, Kerbel RS, Kelloff GJ et al. Rationale for biomarkers and surrogate end points in mechanism-driven oncology drug development. Clin. Cancer Res. 10 (11), 3885-3896 (2004).

19 Zhang H, Li X-J, Martin DB, Aebersold R. Identification and quantification of $\mathrm{N}$-linked glycoproteins using hydrazide chemistry, stable isotope labeling and mass spectrometry. Nat. Biotechnol. 21(6), 660-666 (2003).
20 Nilsson J, Rüetschi U, Halim A et al. Enrichment of glycopeptides for glycan structure and attachment site identification. Nat. Methods 6(11), 809-811 (2009).

21 Xu Y, Wu Z, Zhang L et al. Highly specific enrichment of glycopeptides using boronic acid-functionalized mesoporous silica. Anal. Chem. 81(1), 503-508 (2008).

22 Liu T, Qian W-J, Gritsenko MA et al. Human plasma $\mathrm{N}$-glycoproteome analysis by immunoaffinity subtraction, hydrazide chemistry, and mass spectrometry. J. Proteome Res. 4(6), 2070-2080 (2005).

23 Schiess R, Wollscheid B, Aebersold R. Targeted proteomic strategy for clinical biomarker discovery. Mol. Oncol. 3(1), 33-44 (2009).

24 Yang S, Li Y, Shah P, Zhang H. Glycomic analysis using glycoprotein immobilization for glycan extraction. Anal. Chem. 85(11), 5555-5561 (2013).

25 Dube DH, Bertozzi CR. Glycans in cancer and inflammation-potential for therapeutics and diagnostics. Nat. Rev. Drug Discov. 4(6), 477-488 (2005).

26 Sethi MK, Hancock WS, Fanayan S. Identifying N-glycan biomarkers in colorectal cancer by mass sp ectrometry. Acc. Chem. Res. 49(10), 2099-2106 (2016).

27 Yang S, Höti N, Yang W et al. Simultaneous analyses of $\mathrm{N}$-linked and O-linked glycans of ovarian cancer cells using solid-phase chemoenzymatic method. Clin. Proteomics 14(1), 3 (2017).

28 Royle L, Campbell MP, Radcliffe CM et al. HPLC-based analysis of serum $\mathrm{N}$-glycans on a 96-well plate platform with dedicated database software. Anal. Biochem. 376(1), 1-12 (2008).

29 Qiu Y, Patwa TH, Xu L et al. Plasma glycoprotein profiling for colorectal cancer biomarker identification by lectin glycoarray and lectin blot. J. Proteome Res. 7(4), 1693-1703 (2008).

30 Drake RR, Schwegler EE, Malik G et al. Lectin capture strategies combined with mass spectrometry for the discovery of serum glycoprotein biomarkers. Mol. Cell Proteomics 5(10), 1957-1967 (2006)

31 Hägglund P, Bunkenborg J, Elortza F, Jensen ON, Roepstorff P. A new strategy for identification of $\mathrm{N}$-glycosylated proteins and unambiguous assignment of their glycosylation sites using HILIC enrichment and partial deglycosylation. J. Proteome Res. 3(3), 556-566 (2004).

32 Zhou W, Wang PG, Ogunsola OA, Kraeling ME. Rapid determination of hexapeptides by hydrophilic interaction LC-MS/MS for in vitro skin-penetration studies. Bioanalysis 5(11), 1353-1362 (2013).

33 Mai PL, Wentzensen N, Greene MH. Challenges related to developing serum-based biomarkers for early ovarian cancer detection. Cancer Prev. Res. 4(3), 303-306 (2011).

34 Hanash S, Taguchi A. The grand challenge to decipher the cancer proteome. Nat. Rev. Cancer 10(9), 652-660 (2010). 